doi:10.1017/S0022215116007398

\section{ID: IP243}

Management strategies of glomus jugular tumor

Presenting Author: Yasheng Yuan

Yasheng Yuan ${ }^{1}$, Fanglu $\mathrm{Chi}^{2}$, Qiang $\mathrm{Du}^{2}$, Weidong Zhao ${ }^{2}$, Chunfu Dai ${ }^{2}$, Jing Wang ${ }^{2}$, Bing Chen ${ }^{2}$

${ }^{1}$ EYE \& ENT Hospital of Fudan University,

${ }^{2}$ Department of Otology and Skull Base

Surgery, EYE \& ENT Hospital of Fudan

University, Shanghai 200031

Learning Objectives: Facial nerve management in the surgery Internal carotid artery management in the surgery Intracranial vascular bypass

As for the most challenging surgery of the lateral skull base, glomus jugular tumor resection is now safely controlled and managed. In this study we focused on the the strategies of facial nerve and internal carotid artery management during glomus jugular tumor surgery, especially for the $\mathrm{C}$ and $\mathrm{D}$ stage tumor in which the internal carotid artery was severely involved and could not be easily seperated. Furthermore we tried the extra-intracranial vascular bypass when the internal carotid artery could not be saved in the surgery, by which the mortality and intracranial complications were greatly reduced.

doi:10.1017/S0022215116007404

\section{ID: IP244}

Optotensometry - Developement of an optic method for measuring tubal function by tympanic membrane movement in a middle ear model

\section{Presenting Author: Thorsten Zehlicke}

Thorsten Zehlicke ${ }^{1}$, Hans Georg Fischer ${ }^{2}$, Mark Stamer ${ }^{3}$, Jörg Müller ${ }^{4}$, Hans Wilhelm Pau

${ }^{1}$ Armed Forces Hospital/University of Hamburg,

${ }^{2}$ Armed Forces Hospital/ University of

Hmaburg, ${ }^{3}$ HamburgUniversity of Technology,

Institute of Micro System Technology, ${ }^{4}$ Hamburg

University of Technology, Institute of Micro

System Technology, ${ }^{5}$ University of Rostock, ENT

Department

\section{Learning Objectives:}

Introduction: The amount of pressure in the middle ear depends mainly on the function of the Eustachian tube. Currently there are no continuous recording techniques measuring Eustachian tube function in clinical context and under physiological conditions over extended periods of time.

Purpose: In this paper we investigate the suitability of an active optic triangulation method on the basis of a projected laser-point-pattern in measuring tympanic membrane movement during pressure variations in a middle ear model.

Material and Methods: For projection we used a green semiconductor laser with an output of $1 \mathrm{~mW}$ and a diffractive optical element (DOE). As our measured object we used purple latex-foil (Kimberley-Clark ${ }^{\circledR}$ ), fixed airtight on the cut-off end of a $2 \mathrm{ml}$ syringe-tube. The movement of the foils was measured by an active optic triangulation method. To simulate pathological variations of the tympanic membrane we prepared the latex-foils in specific ways. One foil was perforated and then covered again (simulating tympanic membrane perforation), another one was partly strengthened by sticking a piece of thick, hard paper to it from the inside (simulating calcification).

Results: The test-setup, as well as the appliance of pressure-changes worked fine and measurement of foil movement in all the modified foil surfaces was possible. This shows that it is possible to record foil-movement with this system even in tympanic membranes with pathological variations. Recording tympanic membrane movements in the temporal bone model need a different set up of the measuring system.

Conclusions: In the course of this study we were able to show that it is possible to assess and record foil movement and tympanic membrane movements using a system of optic triangulation and to simulate different tympanic membrane pathologies. This could be used both in ENT medicine, as well as in aviation and diving medicine.

doi:10.1017/S0022215116007416

\section{ID: IP245}

Congenital Cholesteatoma of middle ear in children (14 cases experience)

Presenting Author: Jie Zhang

Jie Zhang, Jinsheng Hao, Min Chen, Wei Liu, Yang Yang, Xin Ni, Jun Zheng

Beijing Children's Hoapital, Capital Medical University

Learning Objectives:

Objective: To investigate the clinical features of Congenital Cholesteatoma (CC) of middle ear in children and provide methods for early diagnosis.

Methods: A retrospective chart review of 94 patients with a diagnosis of middle ear cholesteatoma, in Beijing Children's Hospital, between 2009 and 2015 was carried on. 14 patients with $\mathrm{CC}$ were identified using the criteria proposed by Levenson and Pariser, and were divided into two groups according to the course of disease. The main complains, diagnostic methods and Potsic's stage of temporal bone CT findings were recorded.

Results:

1) $11 / 14$ (78.57\%) patients with CC were boys, with a median age of 7.20 years (from 3.33 to 10.17 years). 
2) Hearing loss $(13 / 14,92.86 \%)$ was the most common complain. Finding methods included hearing test and CT scan $(11 / 14,78.57 \%)$, tympanotomy $(2 / 14,14.28 \%)$ and otoscopic examination( $1 / 14,7.14 \%)$.

3) The preoperative Air-Bone conduction threshold Gap (ABG) was $40.46 \pm 8.86 \mathrm{~dB}$. According to the course of disease, patients with $\mathrm{CC}$ were divided into two groups (A 6/14, B 8/14). There was no significant difference in preoperative ABGs $(38.10 \pm 7.43 \mathrm{~dB} \& 42.09 \pm 9.96 \mathrm{~dB}, \mathrm{P}=$ 0.427 ).

4) According to Potsic's stage among $\mathrm{A}, \mathrm{B}$ groups $(\mathrm{P}=$ 0.043 ).

5) Modified canal wall up mastoidectomy was the preferred procedure and $11 / 14(78.57 \%)$ patients had this surgery done. In 6 patients followed up, the difference between pre-ABG and post-ABG(36.26 $\pm 5.56 \mathrm{~dB} \&$ $21.70 \pm 3.80 \mathrm{~dB}, \mathrm{P}=0.004)$ was significant.

Conclusion: $\mathrm{CC}$ of middle ear in children happened more in boys than in girls. Hearing loss was the most common complaint. The shorter was the course of disease, the less damage of the structure of middle ear would be. Hearing test, CT, tympanotomy and otoscopic examination were usually used for diagnosis.

doi:10.1017/S0022215116007428

\section{ID: IP246}

Bony Meatoplasty with Cartilage

Reconstruction for External Auditory

Canal Cholesteatoma: A Minimal Invasive

Approach

\section{Presenting Author: Ke Zhang}

Ke Zhang, Tao Pan, Qingsong Liu, Jia Ke, Shaoxing Zhang, Furong Ma

\section{Peking University Third Hospital}

\section{Learning Objectives:}

Objective: To assess the outcome and advantage of bony meatoplasty with cartilage reconstruction (BMCR) for stage IV(M) external auditory canal cholesteatoma (EACC) with specific invasion in mastoid cavity.

Methods: Retrospective review of six cases of stage IV(M) EACC. Three cases followed by traditional canal-walldown tympano-mastoidectomy. The other three underwent cartilage reconstruction of bony meatoplasty without radical mastoidectomy.

Results and Conclusion: The preoperative symptoms of all six patients were otalgia, ear fullness and hearing loss. All HRCT demonstrated external auditory canal (EAC) lesions invasion into mastoid cavity. The follow up was between 21 months to 54 months and no recurrence occurred on any of the six patients. It was observed that the patients underwent bony meatoplasty with cartilage reconstruction could also achieve the result of eradicate the disease process. Moreover, compared with the traditional radical operation, BMCR was a minimal invasive approach that maintained the normal structure of EAC and shortened the healing time. The patients underwent BMCR also demonstrated better hearing and quality of life.

doi:10.1017/S002221511600743X

\section{ID: IP247}

Influence of cystic tumor degeneration on management strategy in vestibular schwannoma

\section{Presenting Author: Zhihua Zhang}

Zhihua Zhang, Zirong Huo, Qi Huang, Zhaoyan Wang, Jun Yang, Hao Wu

Department of Otolaryngology Head \& Neck Surgery, Xinhua Hospital Shanghai Jiaotong University School of Medicine, Shanghai Key Laboratory of Translational Medicine on Ear and Nose diseases, Ear Institute Shanghai Jiaotong University, Shanghai 200092, China

\section{Learning Objectives:}

Objective: In this study, we focused on the influence of cystic tumor degeneration on management strategy of vestibular schwannoma.

Methods: The patients with vestibular schwannomawhowere operated at our center from 2006 January to 2013 December were retrospectively analyzed. There were 96 patients having sproradic cystic vestibular schwannomas, recognized by the presence of cystic components both on the preoperative magnetic resonance imaging and intraoperatively, were included. And 96 random cases with solid vestibular schwannomas were used as a control group. The clinical, operative feature and surgical outcomes were reported.

Results: Cystic vestibular schwannomas are associated with rapid growth, worse hearing level $(94.8 \%$ of patients with hearing level in class $\mathrm{C}$ or D) and more frequent onsets of suddent hearing loss than solid tumor. The longterm good facial nerve function rate in cystic tumor is worse than that in solid tumor because of strong adhesion between tumor capsule and facial nerve $(30.2 \%$ vs $44.8 \%, \mathrm{p}=0.037)$. There was no significant difference in complications, mortality and recurrence.

Conclusion: Surgical resection should be the prefer management strategy for cystic vestibular schwannomas. Physician should inform patient with cystic tumor. In case of difficult dissection in peripheral thin wall cystic tumor, near total tumor resection is suggested for protection of facial nerve function and quality of life.

\section{doi:10.1017/S0022215116007441}

\section{ID: IP248}

Hearing restoration for adults with vestibular schwannoma in the onlyhearing ear: ipsilateral, contralateral or bilateral cochlear implantation?

Presenting Author: Zhihua Zhang 